\title{
A Korean Part-of-Speech Tagging System Using Resolution Rules for Individual Ambiguous Word
}

\author{
Young-Min Ahn ${ }^{1}$, Seung-Eun Shin ${ }^{2}$, Hee-Geun Park ${ }^{1}$ \\ Hyungsuk $\mathrm{Ji}^{3}$, and Young-Hoon $\mathrm{Seo}^{1}$ \\ ${ }^{1}$ School of Electrical \& Computer Engineering, Chungbuk National University, Korea \\ nlpmania@paran.com, pinetree@nlp.chungbuk.ac.kr, \\ yhseodchungbuk.ac.kr \\ ${ }^{2}$ BK21 Chungbuk Information Technology Center, Chungbuk National University, Korea \\ seshin@nlp. chungbuk.ac.kr \\ ${ }^{3}$ School of Information \& Communication Engineering, Sungkyunkwan University, Korea \\ jihyungsuk@skku.edu
}

\begin{abstract}
In this paper we present a Korean part-of-speech tagging system using resolution rules for individual ambiguous word. Our system resolves lexical ambiguities by common rules, rules for individual ambiguous word, and statistical approach. We built resolution rules for each word which has several distinct morphological analysis results with a view to enhancing tagging accuracy. Statistical approach based on Hidden Markov Model (HMM) is applied for ambiguous words that are not resolved by the rules. The experiment on the test set shows that the part-of-speech tagging system has high accuracy and broad coverage.
\end{abstract}

Keywords: Part-of-Speech, Tagging, Resolution Rules.

\section{Introduction}

Morphological analyzer is the basic module for most natural language processing systems such as natural language understanding, information retrieval, and machine translation. Morphological analyzer analyzes all possible combination of morphemes for a given word and generates one or more analysis results for that word. In general about $30 \%$ of Korean words have two or more analysis results. Resolving these ambiguities is thus vital to build reliable natural language processing system. Part-ofspeech tagging is the module to resolve such morphological ambiguities. Conventional part-of-speech tagging approaches are classified into statistical approach[1], rule-based approach[2], and hybrid approach [3].

Hidden Markov Model (HMM) has been the mainstay of the statistical model for part-of-speech tagging. It selects a sequence of part-of-speech tags with the highest probability from words or morphemes around an ambiguous word. Rule-based approach resolves ambiguities by predefined rules. Its major advantage is high accuracy but unlike statistical approach it has narrow coverage. Therefore pure rulebased approach is scarcely used in resolving morphological ambiguities. Hybrid 
approach uses both rules and statistical information with a view to achieving high accuracy and broad coverage. Most Korean part-of-speech tagging systems adopt this approach.

This paper presents a part-of-speech tagging system for Korean. The system uses resolution rules for individual ambiguous words to get high accuracy. Each rule has syntax and semantic information for an ambiguous word and words around it. Our system applies common rules to test set followed by resolution rules and statistical tagging.

\section{Building Information for Disambiguation}

\subsection{Common-Rules}

Common-rules are ones for idioms and phrases of common use such as " 할 수 있다 (can)"(halq swu issta), “ 할 수 없다 (cannot)"(halq swu epsta), " 하지 않을 수 없다 (must)"(haci anh.ul swu epsta). In the first stage common-rules are applied to resolve ambiguities of words. For example the tags of “수” (swu) in above expressions are determined as an incomplete noun.

\subsection{Resolution Rules for Individual Ambiguous Word}

In this study, "an ambiguous word" is a word that has two or more distinct morphological analysis results. Ambiguous words was collected and the rules for each ambiguous word was built from a large corpus. 330 ambiguous words which amount to $50 \%$ of total ambiguous words were chosen. Morphemes, part-of-speech tags, word senses, positions, and/or other information of each ambiguous word and words around it were encoded into rules to resolve the ambiguous word. Some rules are shown in Fig.1.

\begin{tabular}{|c|c|c|c|c|}
\hline & & & tag & meaning \\
\hline \multirow[t]{3}{*}{ 있는 } & $/ ! e_{-} @$ & 있/pa+는/etm & $\mathrm{e}_{-}$ & ending \\
\hline & $\begin{array}{l}/ e_{-}+/ j x \\
a\end{array}$ & 있/pa+는/etm & etm & adnominal ending \\
\hline & le_@ & 있/px+는/etm & $\mathrm{pa}$ & adjective \\
\hline \multirow[t]{2}{*}{ 이 } & @ /pa & 이 $/ \mathrm{mm}$ & $\mathrm{pv}$ & verb \\
\hline & (a)/nc & 이 $/ \mathrm{mm}$ & $\mathrm{mm}$ & indeclinable adj. \\
\hline \multirow[t]{3}{*}{ 있었다 } & /!e_@ & 있/pa+었/ep+다/ef & ep & prefinal ending \\
\hline & /e_+/jx@ & 있/pa+었/ep+다/ef & ec & $\begin{array}{l}\text { conjunctive } \\
\text { ending }\end{array}$ \\
\hline & /e_@ & 있/px+었/ep+다/ef & $\mathrm{np}$ & pronoun \\
\hline 그는 & all & 그 $/ \mathrm{np}+$ 는 $/ \mathrm{jx}$ & nc & common noun \\
\hline \multirow[t]{3}{*}{ 남아 } & [열혈]/nc@ & 남아/nc & ef & final ending \\
\hline & (a) [선호]/nc & 남아/nc & $\mathrm{jx}$ & auxiliary particle \\
\hline & default & 남/pv+아/ec & (a) & ambiguous word \\
\hline
\end{tabular}

Fig. 1. An example of resolution rules for individual ambiguous word 


\subsection{Statistical Information}

Information on tag sequences and a word itself were extracted from the tagged corpus. The unit of 3 words information was also extracted. Table 1 shows an example of statistical information extracted in this manner.

Table 1. Example of statistical information

\begin{tabular}{|c|c|c|c|c|c|}
\hline \multicolumn{3}{|c|}{ Information about a word, “있는”(yitnuen) } & \multicolumn{3}{|c|}{$\begin{array}{l}\text { Information about tag sequences, } \\
\text { "nc+co+ec" }\end{array}$} \\
\hline $\begin{array}{l}\text { previous tag } \\
\text { sequences }\end{array}$ & $\begin{array}{c}\text { next tag } \\
\text { sequences }\end{array}$ & frequency & $\begin{array}{l}\text { previous tag } \\
\text { sequences }\end{array}$ & $\begin{array}{c}\text { next tag } \\
\text { sequences }\end{array}$ & frequency \\
\hline $\mathrm{nc}+\mathrm{jc}$ & $\mathrm{nb}+\mathrm{co}+\mathrm{ef}$ & 20 & $\mathrm{~mm}$ & , & 6 \\
\hline $\mathrm{nc}+\mathrm{jc}$ & $\mathrm{nb}+\mathrm{co}+\mathrm{ef}+\mathrm{jx}$ & 4 & $\mathrm{~mm}$ & $?$ & 1 \\
\hline $\mathrm{nc}+\mathrm{jc}$ & $n b+c o+e p+e f$ & 2 & $\mathrm{~mm}$ & EOS & 1 \\
\hline $\mathrm{nc}+\mathrm{jc}$ & $\mathrm{nb}+\mathrm{jc}$ & 17 & $\mathrm{~mm}$ & mag & 8 \\
\hline $\mathrm{nc}+\mathrm{jc}$ & $n b+j x$ & 11 & $\mathrm{~mm}$ & $\mathrm{nc}$ & 1 \\
\hline $\mathrm{nc}+\mathrm{jc}$ & $\mathrm{nc}$ & 33 & $\cdots$ & $\cdots$ & \\
\hline
\end{tabular}

\section{Experiment}

For evaluation, ETRI corpus and Sejong corpus were used for the experiment with sentences. Table 2 shows the information of the test corpus. The rate of ambiguity in the table means the average number of morphological analysis results of ambiguous words. First, we apply common rules to an ambiguous word. Rules for individual ambiguous words are applied subsequently when the ambiguity is not resolved. First matching rule is applied to an ambiguous word when the conditions of two or more rules are satisfied. Statistical information is applied finally when the ambiguity is not resolved. Table 3 shows the result of tagging with the rules only, tagging with only statistical information, and tagging with both.

In Table 3, the average rate of correctness with rules only is 88.68 and resolution accuracy by rules is almost $100 \%$; the average rate of correctness with statistical information is $93.82 \%$. These results are comparable to the best performance of conventional Korean statistical part-of-speech system.

Table 2. The information of corpus for evaluation

\begin{tabular}{cccc}
\hline Corpus & $\begin{array}{c}\text { The total number of } \\
\text { words }\end{array}$ & $\begin{array}{c}\text { The number of } \\
\text { ambiguous words }\end{array}$ & $\begin{array}{c}\text { The rate of } \\
\text { ambiguity }\end{array}$ \\
\hline ETRI corpus & 12357 & 4836 & 2.66 \\
Sejong corpus & 4911 & 1761 & 2.74 \\
Total & 17628 & 6597 & 2.70 \\
\hline
\end{tabular}

Table 3 also shows that the average rate of correctness of our system that uses both rules and statistical information is higher than those of systems that use only rules or only statistical information. Furthermore, the accuracy of our system is $96.86 \%$ which 
is higher than any other conventional hybrid tagging systems whose accuracies varies from $93 \%$ to $96 \%$. Our system does not resolve the ambiguities correctly when two or more consecutive words are all ambiguous.

Table 3. The correctness of each tagging

\begin{tabular}{cccc}
\hline Corpus & $\begin{array}{c}\text { Tagging } \\
\text { with rules }\end{array}$ & $\begin{array}{c}\text { Tagging with } \\
\text { statistical } \\
\text { information }\end{array}$ & $\begin{array}{c}\text { Tagging with rules } \\
\text { and statistical } \\
\text { information }\end{array}$ \\
\hline ETRI corpus & $89.67 \%$ & $95.14 \%$ & $97.74 \%$ \\
Sejong corpus & $87.69 \%$ & $92.50 \%$ & $95.98 \%$ \\
Total & $88.68 \%$ & $93.82 \%$ & $96.86 \%$ \\
\hline
\end{tabular}

\section{Conclusion}

In this paper, we described a hybrid Korean part-of-speech tagging system using rules and statistical information. About $50 \%$ of ambiguities are resolved by those rules, and their accuracy is almost $100 \%$. Statistical tagging based on HMM is applied to words which are not resolved by rules. The correctness of our system was $95.98 \%$, while that of statistical tagging only was $93.82 \%$. The system failed to resolve correctly the ambiguities for consecutive ambiguous words.

Acknowledgments. This research was supported by the Ministry of Information and Communication, Korea under the ITRC, IITA-2006-(C1090-0603-0046).

\section{References}

1. Merialdo, B.: Tagging English Text with a Probabilistic Model. Computational Linguistics 20(2) (1994) 155-171

2. Brill, E.: Transformation-Based Error-Driven Learning and Natural Language Processing: A Case Study in Part-of-Speech Tagging. Computational Linguistics 21(4) (1995) 543-564

3. Tapanainen, P., Voutilainen A.: Tagging accurately - Don't guess if you know. In: Proceedings of the 7th Conference of the European Chapter of the Association for Computational Linguistics. (1994) 149-156 